\title{
Involuntary muscle spasm expressed as motor evoked potential after olfactory mucosa autograft in patients with chronic spinal cord injury and complete paraplegia
}

\author{
Koichi Iwatsuki $^{1 *}$, Toshiki Yoshimine ${ }^{1}$, Yoshiyuki Sankai ${ }^{2}$, Fumihiro Tajima ${ }^{3}$, Masao Umegaki ${ }^{1}$, \\ Yu-Ichiro Ohnishi ${ }^{1}$, Masahiro Ishihara ${ }^{1}, K_{0}$ Kosi Ninomiya ${ }^{1}$, Takashi Moriwaki ${ }^{1}$ \\ ${ }^{1}$ Department of Neurosurgery, Osaka University Medical School, Osaka, Japan \\ ${ }^{2}$ Faculty of Engineering, Information and Systems, University of Tsukuba, Tsukuba, Japan \\ ${ }^{3}$ Department of Rehabilitation, Wakayama Medical University, Wakayama, Japan

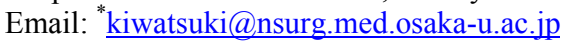

Received 8 July 2013; revised 12 August 2013; accepted 26 August 2013

Copyright (C) 2013 Koichi Iwatsuki et al. This is an open access article distributed under the Creative Commons Attribution License, which permits unrestricted use, distribution, and reproduction in any medium, provided the original work is properly cited.

\begin{abstract}
Object: The efficacy of olfactory mucosa autograft (OMA) for chronic spinal cord injury has been reported. New activity in response to voluntary effort has been documented by electromyography (EMG), but the emergence of motor evoked potential (MEP) reflecting electrophysiological conductivity in the central nervous system, including the corticospinal pathway, after OMA, and the best indications for OMA, have not been clarified. Here, we report the emergence of MEPs after OMA and offer recommendations for appropriate indications based on the presence of involuntary muscle spasm (IMS). We used analysis of MEP to examine the efficacy of OMA for patients with complete paraplegia due to chronic spinal cord injury. To clarify the indications for OMA, we investigated the association of IMS and efficacy of OMA. Methods: Four patients, 3 men and 1 woman, were enrolled. The mean age of the cases was $30.3 \pm 9.5$ years (range, 19 to 40 years). All 4 cases were American Spinal Injury Association (ASISA) grade A. The mean duration from injury to OMA was $95.8 \pm 68.2$ months (range, 17 to 300 months). Samples of olfactory mucosa were removed, cut into smaller pieces, and grafted into the sites of spinal cord lesions after laminectomy. Postoperative subcutaneous fluid collection, postoperative meningitis, postoperative nosebleed, postoperative infection in the nasal cavity, impaired olfaction, neoplastic tissue overgrowth at the autograft site, new sensory disturbance, and involuntary muscle spasm were investigated as safety issues. Improvements in ASIA grade,
\end{abstract}

\footnotetext{
${ }^{*}$ Corresponding author.
}

variations in ASIA scores, EMG, SSEP, and improved urological function were evaluated as efficacy indicators. Results: There were no serious adverse events in this series. In 2 of the 4 cases, an improvement in motor function below the level of injury was recognized. In one, the motor score was 50 until 16 weeks after surgery, and it increased to 52 from 20 weeks after surgery. In the other, the motor score was 50 until 20 weeks after surgery, and it increased to 52 at 24 weeks after surgery with a further increase to 54 at 48 weeks after surgery. The emergence of MEP was recognized in the latter case at 96 weeks after surgery. The other 2 cases had no improvement in ASIA motor score. Both of these cases who showed improvements in the ASIA motor scores exhibited relative IMS compared with those who had no ASIA motor score recovery. Conclusions: We recognized the emergence of MEPs in a case with complete paraplegia due to chronic spinal cord injury after OMA. IMS might be a candidate of indication of OMA.

Keywords: Olfactory Mucosa Autograft; Spinal Cord Injury; Transplantation; Voluntary Movement; Motor Evoked Potential

\section{INTRODUCTION}

The olfactory mucosa is an excellent autologous source of adult neuronal precursor cells. The neurons and sustentacular cells of the olfactory mucosa constantly renew themselves throughout life by proliferation of basal global stem cells [1-3]. Furthermore, the mucosa contains olfactory ensheathing cells, which have previously received much attention for their potential application in 
the repair of spinal cord injuries (SCIs) [4-7]. Recent studies of spinal cord axon regeneration have reported good long-term results using various types of tissue scaffolds [8-10]. Olfactory tissue, which allows autologous transplantation, is easily accessible, and can be obtained by a simple biopsy performed through the external nares [11].

We have previously reported that grafts of the olfactory mucosa are effective in restoring functional recovery in rats following spinal cord transection, with histological evidence of neuronal regeneration [12-14]. Lima et al. performed a clinical trial of olfactory mucosal autograft (OMA) in humans with chronic traumatic SCI and reported restoration of voluntary electromyography (EMG) responses in 15 of 20 cases (75\%) and mean American Spinal Injury Association (ASIA) motor score improvement of $4.95 \pm 7.1$ over a mean follow-up period of 27.7 months. The range of improvement was various, and some cases demonstrated no response to OMA. Therefore, evaluating the possible factors that could predict the efficacy of OMA will be useful. Inclusion criteria for the study by Lima et al. included age, extent of the lesion, and time from the injury, but the authors did not assess the neuronal condition of the severed caudal spinal cord [15]. Furthermore, they did not assess the emergence of motor evoked potential (MEP), which reflects electrophysiological conductivity in the central nervous system, including the corticospinal pathway [16,17], after OMA.

The emergence of involuntary muscle spasm (IMS) after SCI is an indirect measure of the recovery of motor neurons and general motor function [18]. It may indicate plasticity of the spinal cord and the potential for successful regenerative interventions in patients with chronic SCI.

Our pilot study was conducted to examine the emergence of MEP and IMS in OMA patients with chronic $\mathrm{SCI}$ and complete paraplegia.

\section{MATERIAL \& METHODS}

\subsection{Patient Selection and Inclusion Criteria}

This phase I/II nonrandomized, non-controlled, prospective study was approved by the Ethical Committee of the
Osaka University Medical School in Osaka, Japan. All procedures were performed after obtaining written informed consent, which included permission to culture and analyze a biopsy from the tissue to be grafted. Patients were fully aware of the experimental nature of the treatment, the uncertain outcomes, and possible side effects including pain, spasticity, autonomic dysreflexia, worsening of motor or sensory function, infection, and unforeseen adverse events.

Patients who had sustained SCI more than 6 months previously with chronic paraplegia (Table 1) were included. Our rationale for selecting chronic SCI patients (more than 6 months from injury) was to circumvent the spontaneous recovery bias [19]. The other inclusion criteria of this study were generally consistent with those of Lima et al. [15] and comprised ASIA Ggrade A or B; age $\geq 7$ and $\leq 40$ years; presence of a spinal cord lesion $\leq$ $3 \mathrm{~cm}$; absence of significant nasal and paranasal sinus pathology; and absence of additional serious medical problems including respiratory disturbance, brain disease, or psychological disturbance.

Four patients were enrolled in the study, 3 males and 1 female. Demographic data, clinical findings, and imaging/radiological characteristics of the patients are presented in Table 1. The mean age of the patients was 30.3 \pm 9.5 years (range, 19 to 40 years). Injuries were due to traffic accidents in 2 patients, fall in 1 patient, and hemorrhage of unknown origin in 1 patient. The mean maximum lesion size on the vertical axis as measured on both the T1- and T2-weighted MRI was $2.25 \pm 0.57 \mathrm{~cm}$ (range, 1.55 to $2.94 \mathrm{~cm}$ ). All 4 patients were ASISA grade $\mathrm{A}$. The mean time from injury to OMA was $95.8 \pm$ 68.2 months (range, 17 to 300 months).

\subsection{Transplantation Protocol and Surgical Procedure}

Our procedure essentially followed that reported by Lima et al. $[15,20]$. Samples of olfactory mucosa were removed, cut into smaller pieces, and grafted into the spinal cord lesion site after laminectomy. Microbiological examinations of the nasal cavities were performed routinely before surgery and during the operation just prior to transplantation.

Table 1. Summary of demographic and clinical characteristics of 4 patients with olfactory mucosa autografts (OMA).

\begin{tabular}{|c|c|c|c|c|c|c|}
\hline Case No. (years) & Age at OMA & Sex & Months Post-SCI & SCI Level & Length of Lesion & AIS Grade \\
\hline 1 & 40 & Male & 300 & $\mathrm{~T} 4-5$ & 2.2 & A \\
\hline 2 & 19 & Female & 30 & T7-9 & 2.3 & A \\
\hline 3 & 26 & Male & 17 & $\mathrm{~T} 12$ & 1.55 & A \\
\hline 4 & 36 & Male & 36 & $\mathrm{~T} 7-8$ & 2.94 & A \\
\hline
\end{tabular}

Abbreviations: SCI, spinal cord injury; T, thoracic; AIS, ASIA Impairment Scale. 


\subsection{Pre- and Postoperative Rehabilitation}

All patients underwent preoperative rehabilitation (15 $\mathrm{h} /$ week for 4 weeks) and postoperative rehabilitation (15 $\mathrm{h} /$ week for 48 weeks). The preoperative rehabilitation was carried out until immediately prior to the operation and baseline parameters were determined after the preoperative rehabilitation in order to confirm stabilized neurological status. Rehabilitation included standard physical therapy strategies encouraging motor function at and below the lesion to facilitate walking training as soon as possible.

\subsection{Outcome Measures}

Safety and efficacy measures are presented in Table 2. Any improvement in the ASIA grade scale or/and lower extremity motor scores was considered evidence of true gains since all patients had ASIA motor scores of 0 for both legs after the preoperative rehabilitation. The preand postoperative assessment protocol included an ASIA neurological exam, as described in the International Standards for Neurological and Functional Classification of Spinal Cord Injury Patients [21] as well as standard EMG, with recordings taken after patients were asked to move particular muscles, and somatosensory evoked potentials (SSEP), cortically recorded after tibial nerve stimulation; urodynamic studies; full spinal cord MRI scan; otolaryngological evaluation including a general ear, nose, and throat examination, nasal endoscopy, olfactory evaluation, and computed tomography scan of the nose and paranasal sinuses; and psychological assessment. Psychological testing was intended to detect conditions such as active psychosis, major depression, anxiety disorder, severe mood disorder, suicidal behav-

Table 2. Outcome measures.

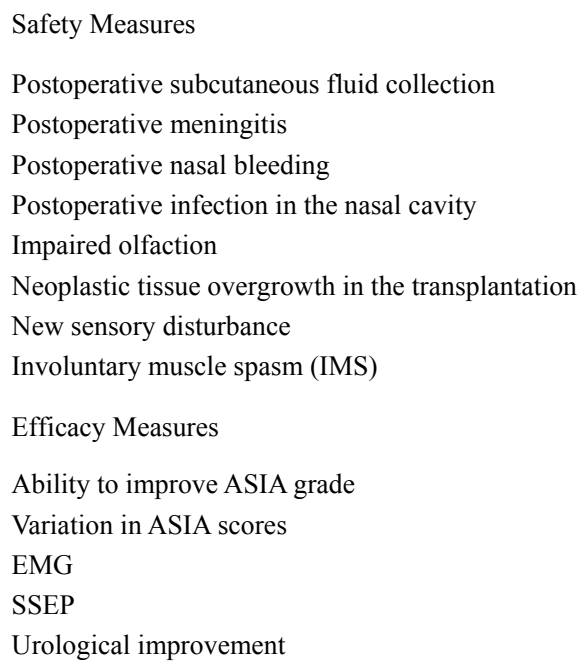

ior, alcohol addiction, drug addiction, low cognitive resources, and unrealistic expectations about treatment results. Pain was assessed via interviews asking the patients to identify painful areas, describe the pain using standard descriptors, and identify temporal aspects of the pain.

We evaluated IMS. There is a variety of tests that attempt to quantify spasticity, but there is no uniformly accepted useful measure $[22,23]$. Our method was simply to note the emergence of IMS on EMG. IMS was evaluated in the bilateral biceps femoris, anterior tibial, flexor digitorum brevis, femoral quadriceps, gluteus maximus, and gastrocnemius muscles. We watched the emergence of IMS while patients rested in the supine position for 3 $\min$ (Figure 1).

MEP response to bifocal transcranial magnetic stimulation (TMS) was evaluated in the bilateral rectus femoris muscles. TMS was performed with a coil $(7 \mathrm{~cm}$ diameter) using a MagPro $\times 100$ (MagVenture A/S, Denmark). Navigation-guided TMS (Brainsight Frameless 1.5; Rogue Research Inc., Montreal, Canada) was used to target the optimal position of each stimulation point. The stimulation hot spot was determined starting about $4 \mathrm{~cm}$ rostral of $\mathrm{Cz}$ (vertex) [24]. Patients who were not able to produce force were asked to exert as much volitional innervation as possible. The duration of the monophasic transcranial single-pulse stimulus was $100 \mu$ s. The sample frequency was $2000 \mathrm{~Hz}$, and a band-pass filter was set at $30 \mathrm{~Hz}$ to $1 \mathrm{kHz}$. TMS was delivered every 5 to $6 \mathrm{~s}$. Three [25] to 5 representative MEPs at the desired stimulus intensity were applied if there was a well-defined response, and up to 10 stimuli were delivered if there was a visible but poorly defined muscle response in order to optimize 3 responses to be stored offline for further analysis [26]. The onset of the fastest response from 4 repeated MEP trials was determined to be the onset latency. The MEP amplitude was calculated from baseline to the negative peak for the largest response out of 4 trials. The intensity of the magnetic stimulus was expressed as a percentage of the maximal stimulator output.

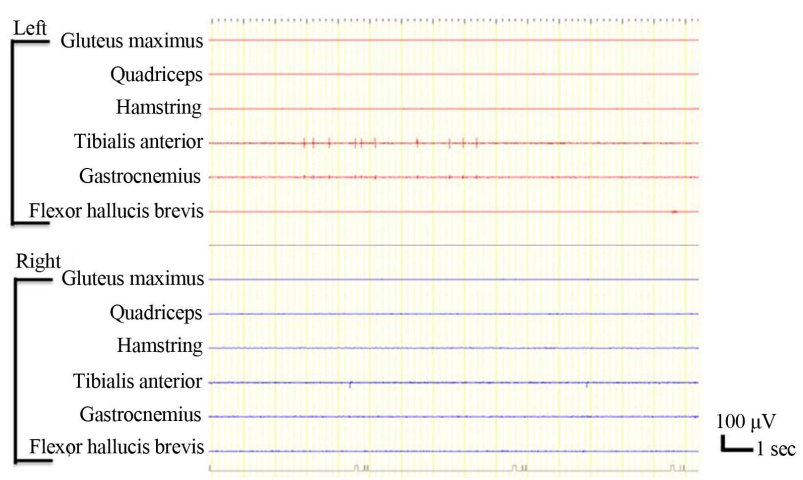

Figure 1. Involuntary muscle spasms were recorded in the left tibialis anterior. 
The patients' neurological findings were evaluated preoperatively and 4, 12, 24, 36, and 48 weeks after OMA (MEP was evaluated additionally at 96 weeks).

\section{RESULTS}

\subsection{Safety}

No serious adverse event occurred in our series. There was no formation of subcutaneous collection of cerebrospinal fluid along the incision in any case. Two cases had postoperative nosebleed treated with tampon gauze and controlled within 7 days. All cases reported impaired sense of smell. Two regained smell within 12 weeks, and the others regained smell within 48 weeks without any further treatments. No case developed meningitis, nasal infection, or neoplasm after surgery. One case reported transient sensory disturbance consisting of pain around the level of injury (Th4 level) at evaluations both 4 and 12 weeks post-surgery. This disturbance resolved spontaneously without any treatment.

\subsection{Efficacy}

\subsubsection{ASIA Scoring Assessments (Motor)}

The data obtained for ASIA motor scores are summarized in Figure 2. No change in ASIA motor score was observed in cases 1 or 3 , but cases 2 and 4 both demonstrated improved motor function below the level of injury. In case 2, the motor score remained at 50 until 16 weeks after surgery and then increased to 52 from 20 weeks until 48 weeks. In case 4 , the motor score was 50 until 20 weeks after surgery and then it increased to 52 at 24 weeks after surgery and further increased to 54 at 48 weeks after surgery.

\subsubsection{ASIA Scoring Assessments}

(Sensory: Pinprick and Light Touch Scores)

No remarkable changes were observed except in case 1 ,

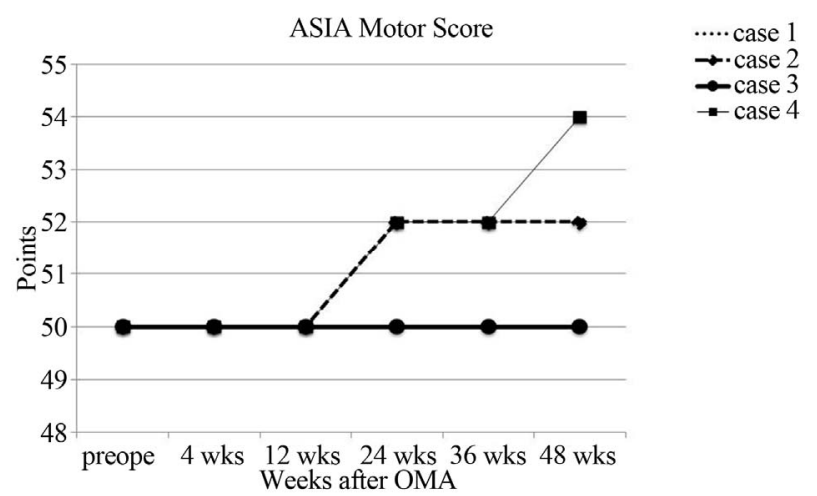

Figure 2. ASIA motor scores. No change in ASIA motor scores was observed in cases 1 and 3, but the score improved from 50 to 52 in case 2 and from 50 to 52 and ultimately 54 in case 4. in whom the score decreased after surgery, from 17 to 2 in response to pinprick and from 15 to 2 in response to light touch.

\subsubsection{Electrophysiological Assessment}

1) EMG assessment (voluntary movement)

New voluntary activity in response to voluntary effort was documented by EMG at 48 weeks after surgery in cases 2 and 4 . In case 2, the activity was recognized in the bilateral tensor fascia lata muscles. In case 4 , the activity was recognized in the bilateral hamstring, anterior tibial, femoral quadriceps, gluteus maximus, and gastrocnemius muscles (Figure 3). There was no activity in response to voluntary effort shown in cases 1 or 3 .

2) SSEP assessment (somatosensory evoked potential)

There were no changes in SSEPs arising from tibial nerve stimulation recorded at the cortical level in any case.

\subsection{Urodynamic Studies}

No case experienced urge to urinate before or after OMA, and all cases remained unable to urinate by themselves.

\subsection{MRI Findings}

MRI 48 weeks after transplantation revealed fairly complete filling of cavities and heterogeneous intensities on T1- and T2-weighted images. Gd-enhanced MRI also showed that the grafts were enhanced heterogeneously. No evidence of neoplastic tissue overgrowth was observed in any case (Figure 4).

\subsection{Involuntary Muscle Spasm}

The evaluation for IMS in each case is documented in Figure 5. In case 3, IMS was not recognized preoperatively or throughout the follow-up period to 48 weeks after OMA (Figure 5). By contrast, in case 4, IMS was recognized preoperatively and throughout the follow-up period to 48 weeks after OMA (Figure 5). Each IMS emergence was calculated as 1 point, as summarized in Figure 6. At the beginning of the follow-up period, case 1 had more IMS points than case 2, but this difference reversed in the middle and latter half of the follow-up period. Thus, we concluded that that IMS could be recognized in cases 2 and 4 rather than the cases 1 and or 3 .

\subsection{Motor Evoked Potentials}

MEPs were not observed before transplantation in any case, but they were recorded at 96 weeks after OMA in case 4 (Figure 7), although not in any other case.

\section{DISCUSSION}

The information about OMA derived from the studies of 

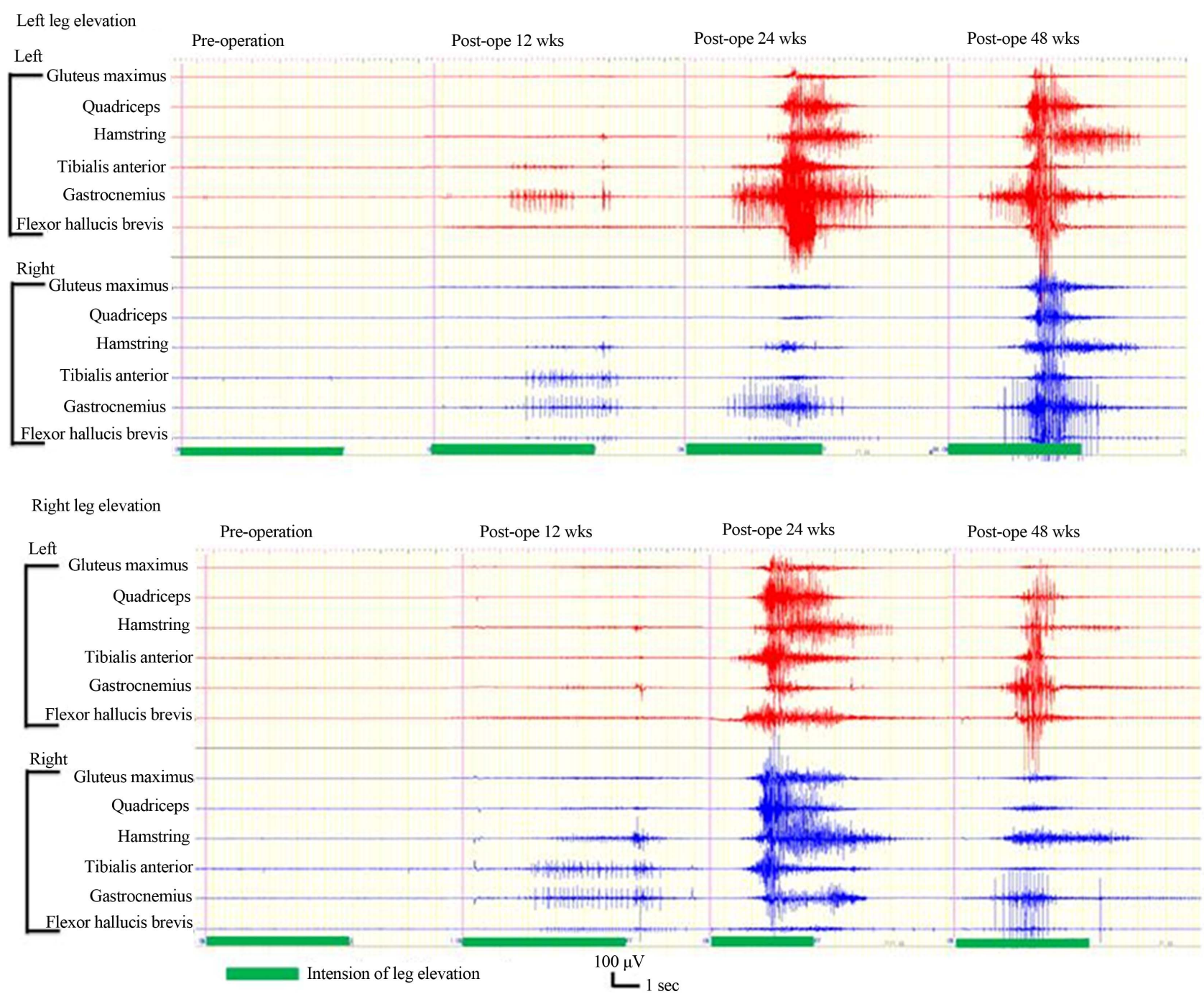

Figure 3. EMG in case 4. New EMG activity in response to voluntary effort was documented at 12, 24, 48 weeks after transplantation. The activity was recognized in the bilateral gluteus maximus, quadriceps, biceps femoris, anterior tibial, gastrocnemius, and abductor hallucis muscles.

Lima et al. is invaluable to basic and clinical researchers investigating regeneration in chronic SCI. Their pioneering work in this field revealed that OMA is fairly safe, feasible, and potentially beneficial $[15,20]$. OMA is advantageous in that it involves transplantation of whole tissue rich in factors that may facilitate neuronal regeneration. We have performed further basic studies of olfactory mucosa transplantation in rats that have supported its feasibility $[12,13]$.

Spinal cord reconstruction using implantation of cells from various sources has gained attention in recent years $[27,28]$. Neuronal stem cells (NSCs) have the potential to differentiate into both neuronal and glial cells, and are therefore prime candidates for cell replacement therapy following CNS injury. NSCs constitutively secrete significant quantities of several neurotrophic factors that act to support host axonal regeneration after SCI [29]. Partial restoration of function after spinal cord contusion has been achieved by injecting neural/glial precursor NSCs differentiated in vitro from mouse embryonic stem cells into the lesion 9 days after injury [30]. However, implantation of NSCs alone did not produce any significant restorative effect because the majority of the NSCs grafted into the spinal cord differentiated into an astrocytic phenotype [29,31]. Although astrocytes can secrete neurotrophic factors and limit the extent of the inflammatory reaction, extensive astroglial scarring within the lesioned area blocks axon growth.

One of the major disadvantages associated with implantation or injection of cells alone is the limited proportion of viable cells surviving at the injury site after the procedure, as cells tend to migrate away from the injury site [32]. To achieve significant functional reconstruction of the spinal cord after SCI, it is necessary to 

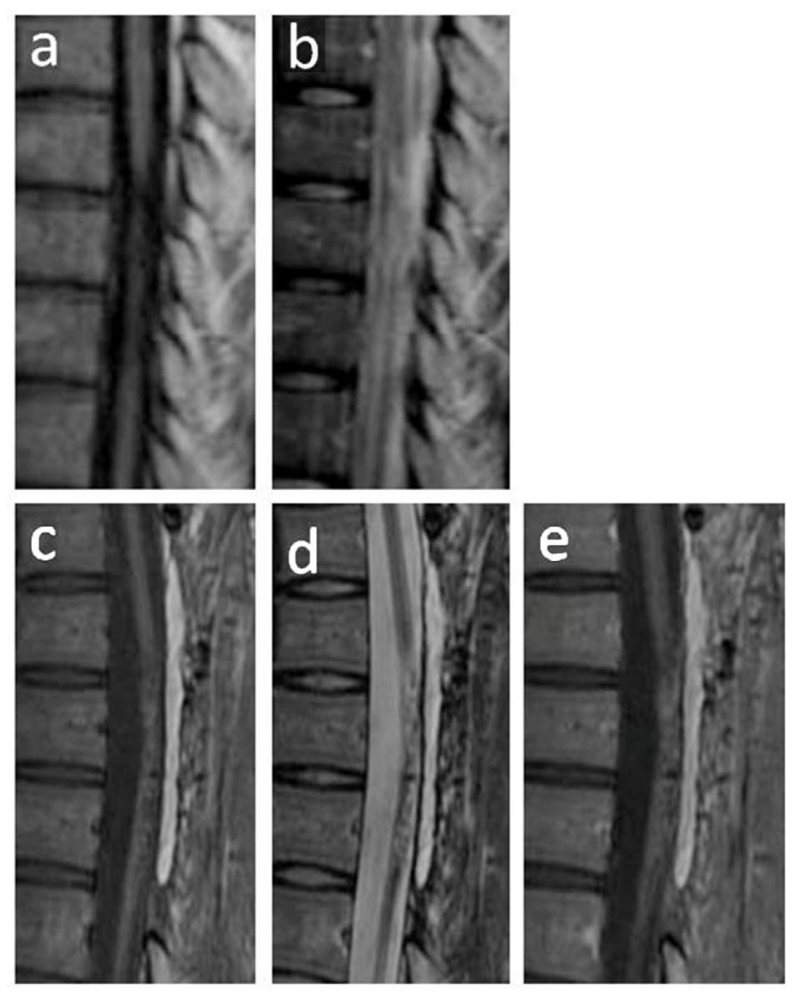

Figure 4. MRI findings in case 2. T1-weighted sagittal image before transplantation showed atrophic change of the thoracic spinal cord (a); T2-weighted sagittal image before transplantation showed an intramedullary high-intensity area (b); MRI scans at 48 weeks after transplantation showed fairly complete filling of cavities and heterogeneous intensity on T1- (c) and T2-weighted images (d); Gd-enhanced MRI showed that the grafts were enhanced heterogeneously (e). No evidence of neoplastic tissue overgrowth was observed.

either populate lesion sites with tissue-specific, regeneration-competent cells or activate endogenous neural progenitor cells to replace or rescue dying cells [33]. The olfactory mucosa seems to be an excellent autologous source of adult neuronal precursor cells. It provides an accessible site for sample biopsy [11] and it contains neurons and sustentacular cells that renew themselves throughout life [1-3] as well as olfactory ensheathing cells that have shown promise in the repair of SCIs [4-7]. These considerations make the nasal mucosa an attractive tissue for potential applications in axonal regeneration. However, while olfactory mucosa may be an ideal tissue for chronic SCI, whether all chronic SCI patients are candidates for OMA remains unclear. Lima et al. applied the following inclusion criteria: ASIA impairment grade A or B [34]; age 18 to 40 years; cervical spinal cord lesion below $3 \mathrm{~cm}$ or thoracic spinal cord lesion below $4 \mathrm{~cm}$; absence of significant nasal and paranasal sinus pathology; and absence of additional serious medical problems, brain disease, or psychological disturbance [20]. Our present clinical trial generally followed these inclusion criteria and additionally investigated IMS. The emergence of IMS after SCI is an indirect measure of recovery of motor neurons and general motor function [18], and it may function as an indicator for the potential success of regeneration therapy in chronic SCI.

In considering the implications of IMS after SCI in our cases, improved motor function below the level of injury was observed in cases 2 and 4, both of whom exhibited relative IMS before OMA and during rehabilitation after OMA. However, no improvement in motor function was observed in cases 1 and or 3, and neither of them had exhibited relative IMS before OMA or during rehabilitation after OMA (Figures 5 and 6). In particular, in case 4, IMS was consistently observed during rehabilitation both before and after OMA (Figure 6), and new voluntary muscle activity was recognized in the bilateral hamstring, anterior tibial, femoral quadriceps, gluteus maximus, and gastrocnemius muscles (Figures $\mathbf{2}$ and 3). By contrast, in case 3, no IMS was observed during rehabilitation before or after OMA (Figures 5 and 6), and no new voluntary muscle activity was observed (Figure 2). The level of injury in this case was Th12. There are motor neurons to generate lower leg movement. These motor neurons were directly injured, and we might not have expected new voluntary muscle activity in such a case. Furthermore, the spinal cord at Th12 is lumbosacral spinal cord, where a central pattern generator (CPG) might exist [4]. The presence of CPG circuitry is thought to be responsible for generation of rhythmic activity within the lumbar cord isolated from brain influence. However, in the absence of descending brain control involved in the initiation of locomotion, CPG activity will be induced by activating afferents from muscles, tendons, and joints by means of peripheral afferent feedback [35]. The CPG in this case might have been injured, and CPG activity might not have been induced by gait training as a peripheral afferent feedback.

We were able to elicit MEPs in case 4. The MEP reflects conductivity in the central nervous system, including the corticospinal pathway $[16,17]$. MEP induced with transcranial magnetic stimulation allows objective assessment of the integrity of human motor circuitry comprising both the corticospinal tract and peripheral motor nerves $[26,36]$. The emergence of MEP in case 4 makes this the first report to indicate the recovery of electrophysiological conductivity after complete chronic SCI by any treatment.

In attempts to perform neuronal repair after SCI, strategies to improve the local environment to promote new neuron formation are essential. However, the condition of motor neurons remaining in the spinal cord should also be considered. Patients may not succeed with OMA without functional motor neurons, and the recov 
Case 1

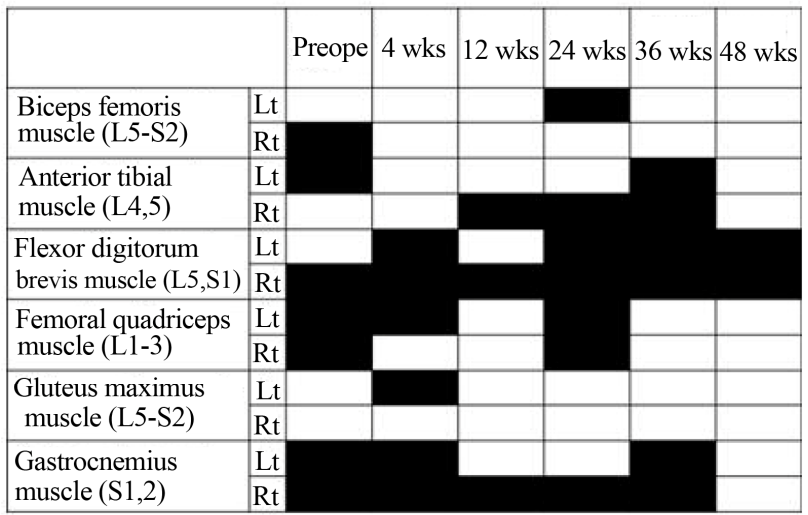

Case 3

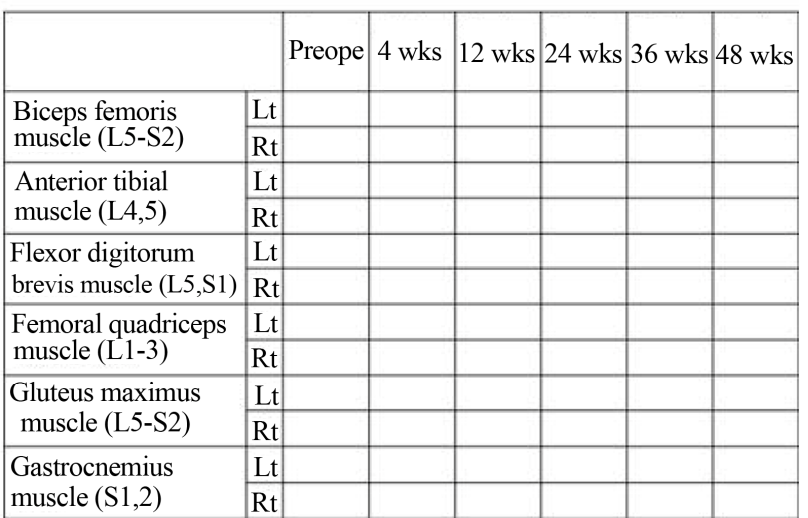

Case 2

\begin{tabular}{|c|c|c|c|c|c|c|c|}
\hline & Preope & 4 wks & $12 \mathrm{wks}$ & 24 wks & $36 \mathrm{wks}$ & $48 \mathrm{wks}$ \\
\hline \multirow{2}{*}{$\begin{array}{l}\text { Biceps femoris } \\
\text { muscle (L5-S2) }\end{array}$} & $\mathrm{Lt}$ & & & & & & \\
\hline & $\mathrm{Rt}$ & & & & & & \\
\hline \multirow{2}{*}{$\begin{array}{l}\text { Anterior tibial } \\
\text { muscle (L4,5) }\end{array}$} & $\mathrm{Lt}$ & & & & & & \\
\hline & $\mathrm{Rt}$ & & & & & & \\
\hline \multirow{2}{*}{$\begin{array}{l}\text { Flexor digitorum } \\
\text { brevis muscle (L5,S1) }\end{array}$} & $\mathrm{Lt}$ & & & & & & \\
\hline & Rt & & & & & & \\
\hline \multirow{2}{*}{$\begin{array}{l}\text { Femoral quadriceps } \\
\text { muscle (L1-3) }\end{array}$} & $\mathrm{Lt}$ & & & & & & \\
\hline & $\mathrm{Rt}$ & & & & & & \\
\hline \multirow{2}{*}{$\begin{array}{l}\text { Gluteus maximus } \\
\text { muscle (L5-S2) }\end{array}$} & $\mathrm{Lt}$ & & & & & & \\
\hline & $\mathrm{Rt}$ & & & & & & \\
\hline \multirow{2}{*}{$\begin{array}{l}\text { Gastrocnemius } \\
\text { muscle }(\mathrm{S} 1,2)\end{array}$} & $\mathrm{Lt}$ & & & & & & \\
\hline & $\mathrm{Rt}$ & & & & & & \\
\hline
\end{tabular}

Case 4

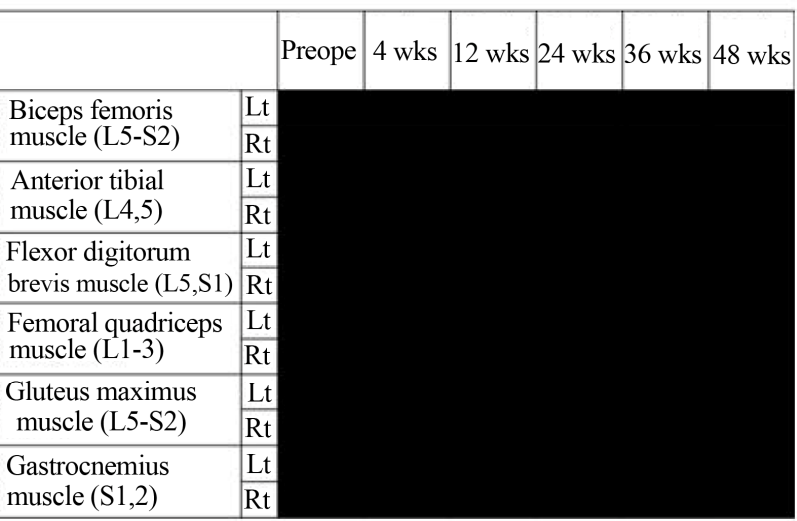

Figure 5. Evaluation for emergence of involuntary muscle spasm (IMS) in 4 cases. Black boxes represent the emergence of IMS. Blanks indicate no IMS. No emergence of IMS was recognized throughout the follow-up period in case 3. On the contrary, IMS was recognized throughout the follow-up period in case 4 . In both cases 1 and 2, moderate IMS was recognized, although more so in case 2 than in case 1 .

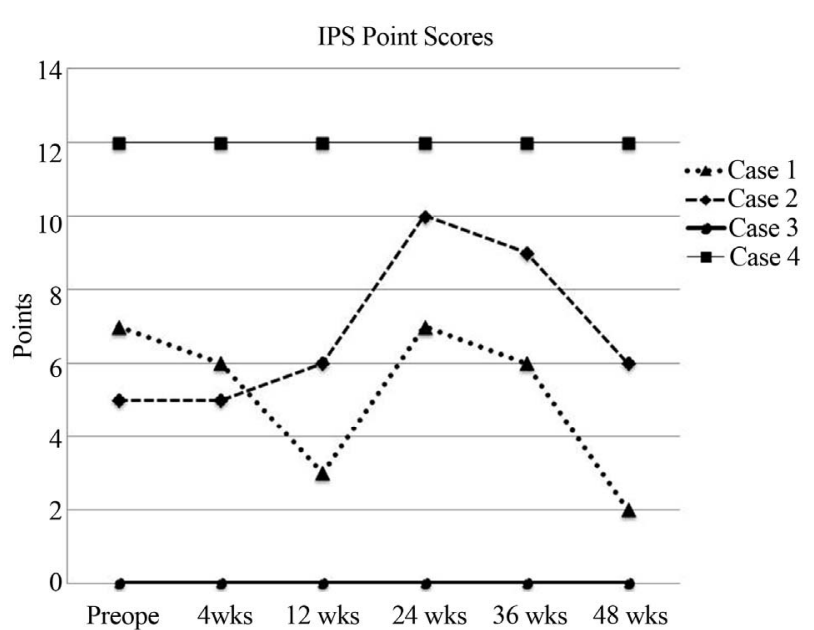

Figure 6. Each emergence of IMS was calculated as 1 point and is summarized in the figure. At the beginning of the follow-up period, case 1 had more points than case 2 , but this difference reversed in the middle and latter parts of the follow-up period. Thus, we concluded that relative IMS could be recognized in cases 2 and 4 rather than in cases 1 and 3 .

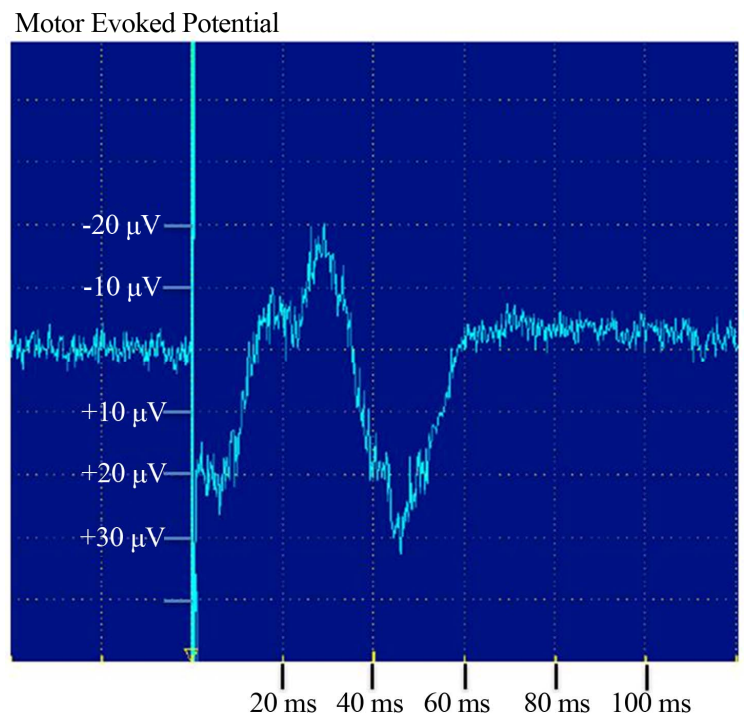

Figure 7. Motor evoked potential (MEP) in response to bifocal transcranial magnetic stimulation (TMS) was evaluated in bilateral rectus femoris muscles. Case 4 exhibited, MEPs in response to TMS. 
ery of motor neurons indirectly indicated by IMS may be a predictor of the success of regeneration therapy in chronic SCI. But we have just only four cases in this study. We have to investigate this in our upcoming clinical trial.

\section{REFERENCES}

[1] Calof, A.L., Bonnin, A. and Crocker, C. (2002) Progenitor cells of the olfactory receptor neuron lineage. Microscopy Research and Technique, 58, 176-188. doi:10.1002/jemt.10147

[2] Cunningham, A.M., Manis, P.B. and Reed, R.R. (1999) Olfactory receptor neurons exist as distinct subclasses of immature and mature cells in primary culture. Neuroscience, 93, 1301-1312. doi:10.1016/S0306-4522(99)00193-1

[3] Roisen, F.J., Klueber, K.M. and Lu, C.L. (2001) Adult human olfactory stem cells. Brain Research, 890, 11-22. doi:10.1016/S0006-8993(00)03016-X

[4] Barnett, S.C. and Riddell, J.S. (2004) Olfactory ensheathing cells (OECs) and the treatment of CNS injury: Advantages and possible caveats. Journal of Anatomy, 204, 57-67. doi:10.1111/j.1469-7580.2004.00257.x

[5] Li, Y., Field, P.M. and Raisman, G. (1997) Repair of adult rat corticospinal tract by transplants of olfactory ensheathing cells. Science, 277, 2000-2002. doi:10.1126/science. 277.5334 .2000

[6] Ramon-Cueto, A. and Valverde, F. (1995) Olfactory bulb ensheathing glia: A unique cell type with axonal growthpromoting properties. Glia, 14, 163-173. doi:10.1002/glia.440140302

[7] Smith, P.M., Lakatos, A. and Barnett, S.C. (2002) Cryopreserved cells isolated from the adult canine olfactory bulb are capable of extensive remyelination following transplantation into the adult rat CNS. Experimental Neurology, 176, 402-406.

doi:10.1006/exnr.2002.7936

[8] Kataoka, K., Suzuki, Y. and Kitada, M. (2004) Alginate enhances elongation of early regenerating axons in spinal cord of young rats. Tissue Engineering, 10, 493-504. doi:10.1089/107632704323061852

[9] Moore, M.J., Friedman, J.A. and Lewellyn, E.B. (2006) Multiple-channel scaffolds to promote spinal cord axon regeneration. Biomaterials, 27, 419-429. doi:10.1016/j.biomaterials.2005.07.045

[10] Stokols, S. and Tuszynski, M.H. (2006) Freeze-dried agarose scaffolds with uniaxial channels stimulate and guide linear axonal growth following spinal cord injury. Biomaterials, 27, 443-451. doi:10.1016/j.biomaterials.2005.06.039

[11] Feron, F., Perry, C. and McGrath, J.J. (1998) New techniques for biopsy and culture of human olfactory epithelial neurons. Archives of Otolaryngology-Head and Neck Surgery, 124, 861-866, doi:10.1001/archotol.124.8.861

[12] Aoki, M., Kishima, H. and Yoshimura, K. (2010) Limited functional recovery in rats with complete spinal cord injury after transplantation of whole-layer olfactory mu- cosa: Laboratory investigation. Journal of Neurosurgery: Spine, 12, 122-130. doi:10.1001/archotol.124.8.861

[13] Iwatsuki, K., Yoshimine, T. and Kishima, H. (2008) Transplantation of olfactory mucosa following spinal cord injury promotes recovery in rats. Neuroreport, 19, 1249-1252. doi:10.1097/WNR.0b013e328305b70b

[14] Nakayama, J., Takao, T. and Kiuchi, H. (2010) Olfactory mucosal transplantation after spinal cord injury improves voiding efficiency by suppressing detrusor-sphincter dyssynergia in rats. Journal of Urology, 184, 775-782. doi:10.1016/j.juro.2010.03.105

[15] Lima, C., Escada, P. and Pratas-Vital, J. (2009) Olfactory mucosal autografts and rehabilitation for chronic traumatic spinal cord injury. Neurorehabilitation and Neural Repair, 24, 10-22. doi:10.1177/1545968309347685

[16] Blight, A.R. (1986) Motor evoked potentials in CNS trauma. Central Nervous System Trauma, 3, 207-214.

[17] Rossini, P.M. (1988 The anatomic and physiologic bases of motor-evoked potentials. Neurol Clin, 6, 751-769,

[18] Murray, K.C., Nakae, A. and Stephens, M.J. (2010) Recovery of motoneuron and locomotor function after spinal cord injury depends on constitutive activity in 5-HT2C receptors. Nature Medicine, 16, 694-700. doi: $10.1038 / \mathrm{nm} .2160$

[19] Ditunno, J.F., Jr. (1992) Functional assessment measures in CNS trauma. Journal of Neurotrauma, 9, S301-305.

[20] Lima, C., Pratas-Vital, J. and Escada, P. (2006) Olfactory mucosa autografts in human spinal cord injury: A pilot clinical study. Journal of Spinal Cord Medicine, 29, 191-203.

[21] Baranowski, P. (2000) Application of the international standards for the neurological and functional classification of spinal cord injuries (the ASIA scale). Ortopedia Traumatologia Rehabilitacja, 2, 31-34.

[22] Granat, M.H., Edmond, P. (1999) The application of air bag technology: An objective clinical measure of involuntary muscle spasm. Spinal Cord, 37, 501-507. doi: $10.1038 / \mathrm{nm} .2160$

[23] Katz, R.T. and Rymer, W.Z. (1989) Spastic hypertonia: Mechanisms and measurement. Archives of Physical Medicine and Rehabilitation, 70, 144-155.

[24] Rossini, P.M., Barker, A.T. and Berardelli, A. (1994) Non-invasive electrical and magnetic stimulation of the brain, spinal cord and roots: Basic principles and procedures for routine clinical application. Report of an IFCN committee. Electroencephalography and Clinical Neurophysiology, 91, 79-92. doi:10.1016/0013-4694(94)90029-9

[25] Cros, D., Soto, O. and Chiappa, K.H. (2007) Transcranial magnetic stimulation during voluntary action: Directional facilitation of outputs and relationships to force generation. Brain Research, 1185, 103-116. doi:10.1016/j.brainres.2007.09.003

[26] Calancie, B., Harris, W. and Brindle, G.F. (2001) Threshold-level repetitive transcranial electrical stimulation for intraoperative monitoring of central motor conduction. Journal of Neurosurgery, 95, 161-168.

[27] Keyvan-Fouladi, N., Raisman, G. and Li, Y. (2003) Functional repair of the corticospinal tract by delayed trans- 
plantation of olfactory ensheathing cells in adult rats. The Journal of Neuroscience, 23, 9428-9434.

[28] Ogawa, Y., Sawamoto, K. and Miyata, T. (2002) Transplantation of in vitro-expanded fetal neural progenitor cells results in neurogenesis and functional recovery after spinal cord contusion injury in adult rats. Journal of Neuroscience Research, 69, 925-933. doi:10.1002/jnr.10341

[29] Han, S.S., Kang, D.Y. and Mujtaba, T. (2002) Grafted lineage-restricted precursors differentiate exclusively into neurons in the adult spinal cord. Experimental Neurology, 177, 360-375. doi:10.1006/exnr.2002.7995

[30] McDonald, J.W., Liu, X.Z. and Qu, Y. (1999) Transplanted embryonic stem cells survive, differentiate and promote recovery in injured rat spinal cord. Nature $\mathrm{Me}$ dicine, 5, 1410-1412. doi:10.1006/exnr.2002.7995

[31] Cao, Q.L., Zhang, Y.P. and Howard, R.M. (2001) Pluripotent stem cells engrafted into the normal or lesioned adult rat spinal cord are restricted to a glial lineage. Experimental Neurology, 167, 48-58. doi:10.1006/exnr.2002.7995

[32] Okada, S., Ishii, K. and Yamane, J. (2005) In vivo imaging of engrafted neural stem cells: its application in evaluating the optimal timing of transplantation for spinal cord injury. The FASEB Journal, 19, 1839-1841.

[33] Stocum, D.L. (2005) Stem cells in CNS and cardiac regeneration. Advances in Biochemical Engineering/Biotechnology, 93, 135-159. doi:10.1007/b99969

[34] Association ASC (2003) ASIA International Standards for Neurological Classification of Spinal Cord Injury, Revised. Spinal Cord Association.

[35] Pinter, M.M. and Dimitrijevic, M.R. (1999) Gait after spinal cord injury and the central pattern generator for locomotion. Spinal Cord, 37, 531-537. doi:10.1038/sj.sc.3100886

[36] Curt, A., Keck, M.E. and Dietz, V. (1998) Functional outcome following spinal cord injury: Significance of motor-evoked potentials and ASIA scores. Archives of Physical Medicine and Rehabilitation, 79, 81-86. doi:10.1016/S0003-9993(98)90213-1 